\title{
Procedures in Practice
}

\section{PROSTATIC BIOPSY}
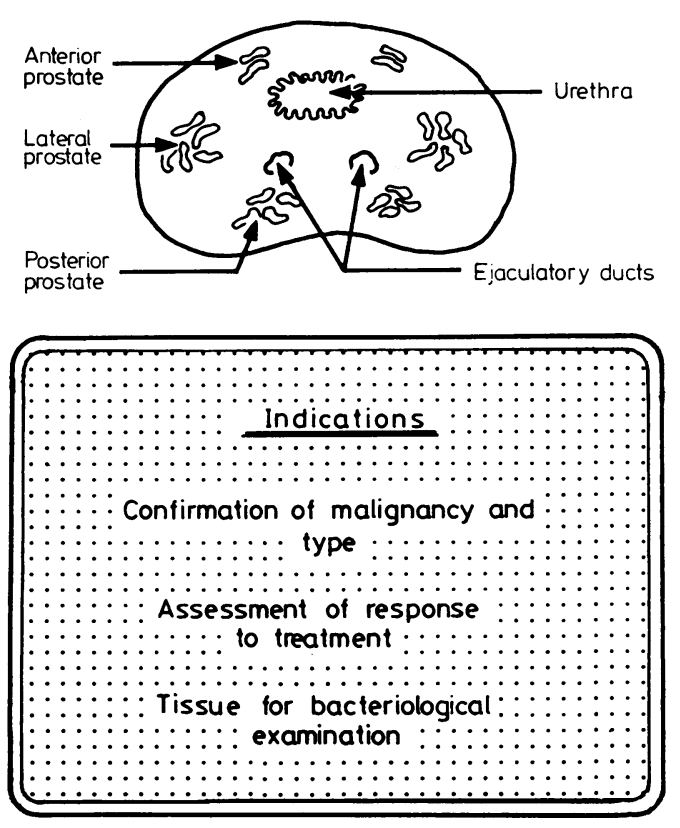

\section{Technique}

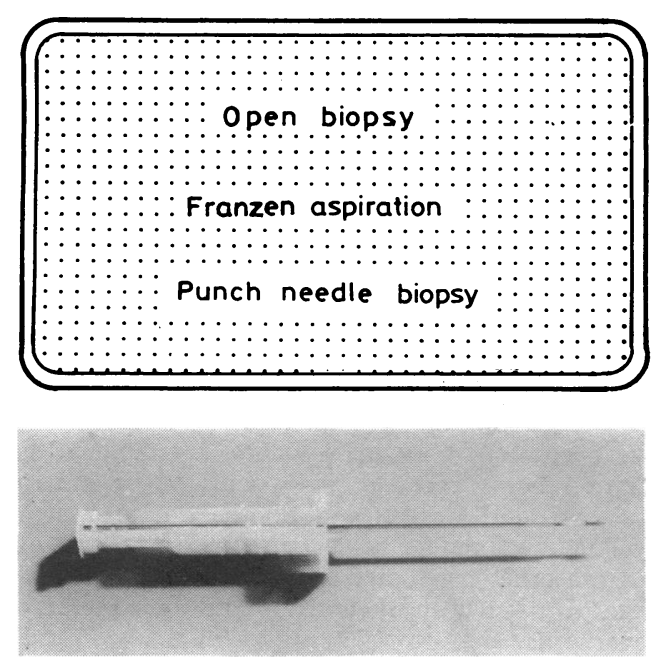

A diagnosis of adenocarcinoma of the prostate based purely on digital examination will be correct in only $70 \%$ of cases. Transitional carcinoma may arise in the prostate, as may more rarely sarcoma. The "fibrous prostate," granulomatous prostatitis, and stones may also result in a prostate that feels firmer than normal. Oestrogens or orchidectomy will be of no benefit in these conditions and may cause harm. Histological or cytological proof of the diagnosis is essential.

Examination of prostatic tissue obtained by biopsy has been used to judge the response of a tumour to either hormonal or cytotoxic manipulation. Culture of the prostate may be useful in difficult cases of prostatitis.

Open biopsy of the prostate by either the perineal or the retropubic route is rarely used in the United Kingdom. Although the perineal approach allows the surgeon the greatest accuracy in obtaining a specimen from a suspicious nodule, the resulting fibrosis may make later total prostatectomy or cystoprostatectomy more difficult. The morbidity is also higher than with the other techniques.

Franzen aspiration of the prostate is the most innocuous of the three techniques but requires the services of a pathologist trained in prostatic cytology and is therefore not always possible.

The most common method of prostatic biopsy practised in the United Kingdom is the punch or needle method using the Tru-Cut needle or the Franklin modification of the Vim-Silverman needle. This provides a core of tissue suitable for routine histological examination. 


\section{Method}
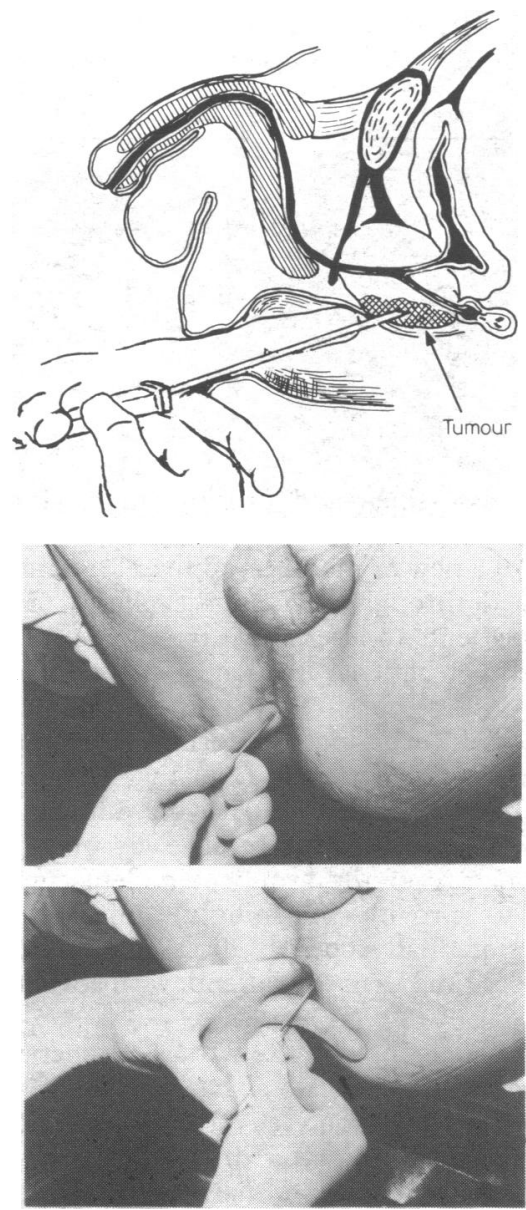

Needle biopsy may be performed as an outpatient procedure, but since penetration of the prostate by the needle causes some discomfort general anaesthesia, local block, or intramuscular pethidine and diazepam are necessary. The patient is placed in either the lithotomy or lateral position with any degree of Trendelenburg thought to be necessary. Very few patients are unsuitable because of ill health or inaccessibility of the prostate.

The biopsy may be performed using either a perineal or transrectal route; these methods are described separately.

Sampling via the perineal route is a semi-sterile technique and requires the perineum to be shaved and the skin cleansed in the usual manner. A tiny incision in the midline is made with a size 15 blade $1.0-1.5 \mathrm{~cm}$ anterior to the anal verge. The biopsy needle is inserted through this incision into the prostate and its course to the area to be sampled estimated by placing a finger in the rectum.

Transrectal biopsy entails passing the needle with the examining finger into the rectum and is therefore a non-sterile technique. To minimise trauma to the anal mucosa the sharp tip of the needle should be protected by the pulp of the finger during insertion. The surgeon then palpates the area of prostate in doubt and the needle is introduced direct through the rectal mucosa.

In both methods tissue is obtained by advancing the trocar and then closing the cannula on to the trocar. With a little practice the trocar may be held firmly by the remaining fingers of the left hand so that removal of the finger from the rectum is not necessary. The tissue is then placed in preservative for histological examination or transport medium for bacteriological examination. Several samples may be obtained at the same time, although this slightly increases the risk of complications.

\section{Complications}

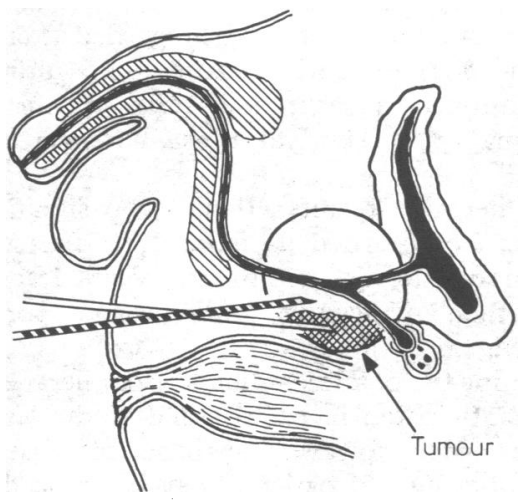

Biopsy failure occurs when no tissue is obtained or the histology of the specimen does not agree with the final diagnosis. The transrectal route has a lower failure rate than the perineal route. This is partly because the operator has a better idea of which portion of the prostate the needle is sampling but also because carcinoma tends to arise in the peripheral part of the gland. This area may be missed when the perineal route is used, although with practice the accuracy of this method should improve. Biopsy failure may also occur when the patient has had a prostatectomy so that only the relatively thin shell of the false capsule remains, or when the tissue is so friable that none can be picked up by the needle.

Rectal bleeding or haematuria may occur after biopsy but is only rarely a problem. Haematuria is not uncommon when biopsy is by the perineal route and may persist for several days, but unless the patient has coagulation problems transfusion is unnecessary.

Infection is the main complication of the transrectal route and may proceed to bacteriogenic shock in approximately $0.5 \%$ of cases. Prophylactic antibiotics will reduce the infection rate but to be of value must be started before the biopsy procedure.

Tumour implantation is the fear in any biopsy procedure but is extremely rare in the case of prostatic needle biopsy and has been reported only in association with the perineal route. 